\title{
Incorporation of CD40 ligand enhances the immunogenicity of tumor-associated calcium signal transducer 2 virus-like particles against lung cancer
}

\author{
WANG XI" ${ }^{*}$ DONG KE* ${ }^{*}$ LONG MIN, WANG LIN, ZUO JIAHUI, LIN FANG, \\ GAO ZHAOWEI, ZHANG ZHE, CHEN XI and ZHANG HUIZHONG \\ Department of Medical Laboratory and Research Center, Tangdu Hospital, Fourth Military Medical University, \\ Xi'an, Shaanxi 710038, P.R. China
}

Received October 12, 2017; Accepted March 5, 2018

DOI: $10.3892 /$ ijmm.2018.3570

\begin{abstract}
The cell surface glycoprotein Trop-2 is overexpressed in various types of cancer, including in lung cancer, and has recently been used as an effective immunotherapeutic target. CD40 ligand (CD40L), a tumor necrosis factor superfamily member, is a promising immune adjuvant. Human immunodeficiency virus (HIV) gag-based virus-like particles (VLPs) are highly immunogenic, and foreign antigens can be incorporated onto their membrane envelope for cancer vaccine development. In the present study, a HIV gag-based VLP strategy and Bac-to-Bac system were utilized to construct Trop-2, CD40L and gag recombinant baculoviruses, which were then used to infect TN5 cells in order to form Trop-2 VLPs or Trop-2-CD40L VLPs. These VLPs were characterized using transmission electron microscopy and western blot analysis methods. VLPs incorporating murine Trop-2 only or incorporating Trop-2 and CD40L were used to immunize C57BL/6 mice. Immunized mice demonstrated high humoral and cellular immunity responses, whereas the Trop-2-CD40L VLPs led to higher immune responses in comparison with Trop-2 only VLPs. Immunization with Trop-2-CD40L VLPs also reduced tumor growth more effectively compared with Trop-2 VLPs. Furthermore, Trop-2-CD40L VLP immunization increased the survival rate of Lewis tumor-bearing mice more significantly when compared with Trop- 2 only VLPs. In conclusion, the present study provided a novel vaccine design by combination of a tumor antigen and an immune adjuvant based on a VLP strategy, which may be potentially applied as
\end{abstract}

Correspondence to: Dr Zhang Huizhong, Department of Medical Laboratory and Research Center, Tangdu Hospital, Fourth Military Medical University, 1 Xinsi Road, Xi'an, Shaanxi 710038, P.R. China E-mail: td_zhz@163.com

${ }^{*}$ Contributed equally

Key words: Trop-2, CD40 ligand, lung cancer, virus-like particles an alternative immunotherapeutic option in the treatment of lung cancer.

\section{Introduction}

The tumor-associated calcium signal transducer 2 (Trop-2) is a type-1 transmembrane protein. Although Trop-2 is overexpressed in a large variety of human epithelial carcinomas (1-5), its expression is minimal or restricted in normal tissues. Notably, elevated expression of Trop-2 is associated with more aggressive disease and poor prognosis in several cancer types (6-8). The study by Jiang et al (9) revealed that, in patients with advanced non-small cell lung cancer (NSCLC), Trop-2 expression was higher in tumor tissues in comparison with that in the tumor-adjacent normal tissues. In addition, the study identified that Trop-2 expression was correlated with the clinicopathological features (9). These previous studies investigating Trop- 2 indicated that it is a novel tumor antigen and may potentially be used as a lung cancer immunotherapy target.

Virus-like particles (VLPs) contain the structure of native virions, however, they are non-infectious due to lack of the virus genome (10-12). Recently, recombinant VLP-based vaccine strategies have been frequently used for novel vaccine development. Various types of VLPs have been designed and produced by utilizing the self-assemble ability of virus capsid and envelope proteins (13-20). Commercialized VLP-based vaccines have been successfully used in the prevention against hepatitis B virus and human papillomavirus (21). The structural polyprotein Pr55gag of the human immunodeficiency virus type I (HIV-1) gag gene directs the viral assemble process, and the unprocessed gag precursor itself is sufficient for the release of non-infectious VLPs. In addition to their self-assembly abilities, Pr55gag-based VLPs are able to deliver foreign polypeptides, and thus can be used both as a direct immunogen and as a carrier for foreign antigens. Two principal strategies have previously been established (22), involving the type I VLPs that are constructed by integrating or fusing small epitopes with the C-terminus of gag polyprotein, as well as the type II VLPs that are constructed by incorporating foreign proteins at the outer particle surface. 
Although the use of VLPs is a promising strategy for vaccine development, identifying approaches to enhance the immunogenicity of VLPs is highly desirable. In the field of tumor immunotherapy, the application of adjuvants is widely recognized. A number of tumor necrosis factor superfamily ligands (TNFSFLs) have been demonstrated to participate in the activation processes of dendritic cells and $\mathrm{T}$ cells, and such molecules include the CD40 ligand (CD40L), CD70, OX40L, GITRL, RANKL, LIGHT, 4-1BBL and BAFF $(23,24)$. In addition, TNFSFLs as immune adjuvants have previously been reported (25). As a member of the TNFSFL family, CD40L is mainly expressed on the surface of activated $\mathrm{CD} 4^{+} \mathrm{T}$ cells, and is able to enhance the humoral and cellular immune responses $(26,27)$. Furthermore, the CD40L/CD40 interaction serves a significant role in B-cell activation, proliferation, differentiation and antibody production (28), whereas binding of CD40L to CD40 induces the expression of costimulatory molecules that reside on antigen-presenting cells. Once these cells are activated, the $\mathrm{CD} 4^{+} \mathrm{T}$ cell responses are augmented by increased cytokine production, while the $\mathrm{CD} 4^{+}$-dependent $\mathrm{CD}^{+} \mathrm{T}$ cells are also activated (29). In the field of DNA vaccine design, fusion of the immune stimulatory molecule CD40L genetically was confirmed to be an effective way to obtain a robust $\mathrm{T}$ cell response (30). In the field of VLP development, Skountzou et al (31) also demonstrated that incorporation of CD40L into VLPs resulted in enhanced immunogenicity of viral antigens by stimulating $\mathrm{T}$ cell responses.

In the present study, the construction and characterization of type II gag VLPs was reported. These VLPs were produced in insect cells and constructed from full-length gag molecules incorporating Trop-2 alone or a combination of Trop- 2 and CD40L adjuvant on the surface of VLPs. First, whether immunization with either of these VLPs was able to generate specific humoral and cellular immune responses against Trop-2 was examined, and whether CD40L-incorporated VLPs generated a higher immune response compared with Trop-2 only VLPs was also examined. Prevention and therapeutic experiments were performed to examine whether VLP immunization may result in reduced tumor growth and increased survival rate of Lewis ${ }^{(\text {Trop-2) }}$ tumor-bearing mice, and whether CD40L-incorporated VLPs exhibited a more efficient tumor inhibition effect when compared with Trop-2 only VLPs. Given the high resistance of lung cancer to conventional treatments and sensitivity to immunotherapy, the present study provided a promising approach using VLPs as a carrier of the novel tumor antigen Trop- 2 and the immune adjuvant CD40L.

\section{Materials and methods}

Cells. Lewis murine lung adenocarcinoma cells were purchased from American Type Culture Collection, (Manassas, VA, USA; ATCC ${ }^{\circledR}$ no. 18188) and cultured in Dulbecco's modified Eagle's medium (DMEM) supplemented with $10 \%$ fetal bovine serum (FBS) (both from Gibco; Thermo Fisher Scientific, Inc., Waltham, MA, USA), at $37^{\circ} \mathrm{C}$ in an atmosphere of $5 \% \mathrm{CO}_{2}$ and $95 \%$ humidified air. In addition, the normal insect cell line TN5 was provided by Dr Ye Jing (The Fourth Military Medical University, Xi'an, China) and were cultured in Sf-900 II (Gibco; Thermo
Fisher Scientific, Inc.) serum-free insect cell culture medium. Full-length murine Trop-2 (mTrop-2) cDNA was cloned into the pcDNA3.1 vector and termed plasmid (p-Trop-2). To generate Lewis cells stably expressing mTrop-2, the p-Trop-2 was transfected into Lewis cells using Lipfectamine 2000 (Invitrogen; Thermo Fisher Scientific, Inc.) at $37^{\circ} \mathrm{C}$. A total of 4-6 h later, the medium was replaced with fresh DMEM and culturing was continued for $24 \mathrm{~h}$ at $37^{\circ} \mathrm{C}$. Positive clones were selected using $700 \mu \mathrm{g} / \mathrm{ml} \mathrm{G} 418$ (Invitrogen; Thermo Fisher Scientific, Inc.), incubated for 14 days at $37^{\circ} \mathrm{C}$, and the transfected cells presenting a stable expression of mTrop-2 were termed as Lewis ${ }^{\text {(Trop-2) }}$.

DNA constructs and preparation of recombinant baculoviruses ( $r B V s)$. Plasmids containing mouse CD40L or HIV gag gene were kindly provided by Dr Ye Ling (Department of Microbiology and Immunology, Emory University, Atlanta, Georgia). CD40L was amplified by the polymerase chain reaction (PCR) method using the following primers: CD40L forward (carrying an EcoRI site), 5'-ccggaa ttcgccacctaatgatagaaacatacagcc-3', and reverse (carrying an XhoI site), 5'-cccgctcgagtcagagtttgagtaagcc-3', with the underlined parts of the sequences indicating the restriction enzyme recognition sites. In addition, gag gene fragments were also obtained by PCR using the following primers: Gag forward (EcoRI site), 5'-ccggattcatgggagatagggtggcagatgtaat-3', and reverse (HindIII site), 5'-cccaagcttttactaactggtctcctccaaagagag aat-3'. The thermocycling conditions were as follows: 1 cycle for $30 \mathrm{~s}$ at $98^{\circ} \mathrm{C} ; 30$ cycles for $30 \mathrm{~s}$ at $65^{\circ} \mathrm{C}$, and $1 \mathrm{~min}$ at $72^{\circ} \mathrm{C}$; and 1 cycle for $10 \mathrm{~min}$ at $72^{\circ} \mathrm{C}$. PCR products were purified using the PrimeSTAR ${ }^{\circledR}$ HS DNA Polymerase (Takara Bio Inc., Otsu, Japan).

A newborn normal female mouse at 0-3 days of age (weight, $1.5 \mathrm{~g}$ ), housed under a controlled temperature $\left(\sim 22^{\circ} \mathrm{C}\right)$ and relative humidity $(40-60 \%)$ conditions with a 12-h light-dark cycle and breast fed, was anesthetized and sacrificed using ether, then the kidney was isolated, and the RNA was extracted using TRIzol reagent (Invitrogen; Thermo Fisher Scientific, Inc.). The full-length mTrop-2 cDNA was then amplified using the reverse transcription-PCR method with the following primers: Trop-2 forward (EcoRI site), 5 '-ccggaattcatggcgaggggcttggatctagcac-3', and reverse (XhoI site), 5'-cccgctcgagctacaagctaggttcgcttctcatctcccc-3'. The thermocycling conditions were 1 cycle for $30 \mathrm{~s}$ at $98^{\circ} \mathrm{C} ; 30$ cycles for $2 \mathrm{~min}$ at $68^{\circ} \mathrm{C}$; and 1 cycle for $10 \mathrm{~min}$ at $72^{\circ} \mathrm{C}$. PCR products were purified using PrimeSTAR ${ }^{\circledR}$ HS DNA Polymerase (Takara Bio Inc.).

Gag, CD40L and Trop-2 genes were cloned into multiple cloning sites of the pFastBacI vector (Invitrogen; Thermo Fisher Scientific, Inc.), respectively. The pFastBacI plasmids containing the gag, Trop-2 or CD40L genes were introduced by transformation into DH10Bac ${ }^{\mathrm{TM}} \mathrm{E}$. coli at $37^{\circ} \mathrm{C}$ for $48 \mathrm{~h}$ (Invitrogen; Thermo Fisher Scientific, Inc.). Following verification of DH10Bac with recombinant bacmid, recombinant bacmid DNA was isolated. The rBVs expressing the gag, Trop-2 or CD40L proteins were generated by transfecting TN5 insect cells with recombinant Trop-2 or CD40L bacmids using Cellfectin II Reagent (Thermo Fisher Scientific, Inc.) at $27^{\circ} \mathrm{C}$ for 4 days, and the culture supernatant containing the $\mathrm{rBv}$ was collected 4 days post transfection. 
$V L P$ production and purification. TN5 cells were infected with rBV gag at a multiplicity of infection (MOI) of 2 in order to release gag VLPs. Next, VLPs containing Trop-2 were produced by co-infecting TN5 cells with rBV gag and rBV Trop-2 at a MOI ratio of 1:5. Chimeric VLPs containing both Trop-2 and CD40L were also produced by coinfecting TN5 cells with rBV gag, Trop-2 and CD40L at a MOI ratio of 1:5:2. The medium containing the VLPs was collected at $72 \mathrm{~h}$ post infection, and the cell debris was clarified by high-speed centrifugation $\left(3,000 \times \mathrm{g}, 30 \mathrm{~min}, 4^{\circ} \mathrm{C}\right)$. Subsequently, the supernatant containing the VLPs was concentrated with a QSM-03SP/50quixstand ${ }^{\mathrm{TM}}$ Benchtop System (GE Healthcare Life Sciences, Little Chalfont, UK) and further purified by ultra-centrifugation at $100,000 \mathrm{x}$ g for $2.5 \mathrm{~h}$ at $4^{\circ} \mathrm{C}$ in an SW-41 rotor (Beckman Coulter, Inc., Brea, CA, USA) through a $15-50 \%$ discontinuous sucrose gradient. The layer containing the purified VLPs was then suspended, washed with phosphate-buffered saline (PBS), ultra-centrifuged at 28,000 rpm for $30 \mathrm{~min}$ and re-suspended in PBS. A BCA assay kit (Thermo Fisher Scientific, Inc. CA, USA) was subsequently used to detect the protein concentration of the VLPs.

Western blot analyses of VLPs. Purified VLPs were characterized by western blot analysis for the presence of Trop-2 and CD40L proteins. Briefly, the VLPs were quantified using a BCA assay kit (cat. no. BCA02; Beijing Dingguo Changsheng Biotechnology Co., Ltd., Beijing, China) according to the manufacturer's protocol, samples of 5,2, and $1 \mu \mathrm{g}$ were loaded per lane and were subjected to $10 \%$ SDS-PAGE, and transferred to a nitrocellulose membrane (Whatman $\mathrm{GmbH}$, Dassel, Germany). The membrane was blocked using $5 \%$ nonfat milk in PBS with $20 \%$ Tween-20 at $4^{\circ} \mathrm{C}$ overnight, and then incubated with polyclonal rabbit anti-HIV gag p24 (1:2,000 dilution; cat. no. ab32352; Abcam, Cambridge, MA, USA), mouse anti-Trop-2 (1:1,000 dilution; cat. no. sc-376181; Santa Cruz Biotechnology, Inc., Dallas, TX, USA) and monoclonal mouse anti-CD40L (1:2,000 dilution; cat. no. MAB4401; R\&D Systems, Inc., Minneapolis, MN, USA) primary antibodies overnight at $4^{\circ} \mathrm{C}$. Then, the membrane was washed three times with PBST for 10 min each. Subsequently, the samples were incubated with horseradish peroxidase (HRP)-conjugated goat anti-rabbit (1:5,000; cat. no. ZDR-5306) or HRP-conjugated goat anti-mouse IgG (1:5,000; cat. no. ZDR-5307) secondary antibodies (both from OriGene Technologies, Inc., Beijing, China) for $1 \mathrm{~h}$ at room temperature. The protein bands were detected with an enhanced chemiluminescence developing reagent (Sigma-Aldrich; Merck KGaA, Darmstadt, Germany). Protein bands were quantified by densitometry using ImageQuant TL 7.0 Image Analysis Software (GE Healthcare Life Sciences).

Transmission electron microscopy (TEM). VLPs were further examined by TEM, which was performed as previously described (32). Briefly, the Trop-2 or Trop-2-CD40L VLPs prepared as described above were negatively stained with $1 \%$ potassium phosphotungstate (cat. no. GZ02536; Beijing Zhongjingkeyi Technology Co., Ltd, Haidian, China) at room temperature for $1 \mathrm{~min}$, washed with PBS for $1 \mathrm{~min}$ at room temperature and then examined using a Hitachi-H7500 TEM system (Hitachi, Ltd., Tokyo, Japan).
Characterization of CD4OL incorporated into VLPS by $B$-cell activation. To measure the B-cell activation, one female C57BL/6 mouse (aged 6 weeks; weight, $20 \mathrm{~g}$ ), housed under a controlled temperature $\left(22^{\circ} \mathrm{C}\right)$ and relative humidity (40-60\%) conditions with a 12-h light-dark cycle and provided ad libitum access to a standard laboratory diet of food and water was sacrificed by cervical dislocation. The spleens were removed and processed into single-cell suspensions in RPMI 1640 supplemented with $2 \mathrm{mM}$ L-glutamine (Invitrogen; Thermo Fisher Scientific, Inc.), 10\% fetal bovine serum (HyClone Systems, Inc., Logan, UT, USA), 100 U of penicillin per $\mathrm{ml}$ and $100 \mu \mathrm{g} / \mathrm{ml}$ streptomycin. The isolated spleen cells were cultured in 48 -well cell culture plates in triplicate, with or without $2 \mu \mathrm{g} / \mathrm{ml}$ VLP stimulation. The cells were then collected 4 days post stimulation, and $10^{6}$ cells were stained with phycoerythrin-conjugated anti-CD69 (1:2,000 dilution; cat. no. MA1-10276; eBioscience; Thermo Fisher Scientific, Inc.) and peridinin chlorophyll protein-conjugated anti-B220 (1:2,000 dilution; cat. no. 45-0452-82; eBioscience; Thermo Fisher Scientific, Inc.) at room temperature for $1 \mathrm{~h}$ in the dark. Cells were subsequently washed with PBS, fixed with $1 \%$ paraformaldehyde and analyzed by flow cytometry (Cytomics FV 500; Beckman Coulter, Brea, CA, USA).

Immunization. A total of 24 pathogen-free female C57BL/6 mice (age, 6-8 weeks old, weight, 20-23 g) kept in pathogen-free environments were purchased from The Fourth Military Medical University Animal Center (Xi'an, China). Mice were housed under a controlled temperature $\left(\sim 22^{\circ} \mathrm{C}\right)$ and relative humidity (40-60\%) conditions with a 12-h light-dark cycle and provided ad libitum access to a standard laboratory diet of food and water. Animal studies were performed according to the current animal experimental protocols approved by the Institutional Review Board of Tangdu Hospital at the Fourth Military Medical University (assigned no. TDLL-201611-25). In order to examine the effect of VLP immunization, female C57BL/6 mice (6-8-week-old) were randomly divided into four groups (6 mice/group), and each mouse was immunized twice with a 2 -week interval by subcutaneous injection with $40 \mu \mathrm{g}$ gag VLPs, Trop-2 VLPs, Trop-2-CD40L VLPs or $100 \mu \mathrm{l}$ PBS, respectively. Retro-orbital bleeding was used to collect blood samples at 2 weeks post each immunization. The serum samples were inactivated at $56^{\circ} \mathrm{C}$ for $30 \mathrm{~min}$ and then stored at $-80^{\circ} \mathrm{C}$. At 1 week after the last vaccination, mice were challenged subcutaneously in the right flank with $1 \times 10^{6}$ Lewis $^{(\text {Trop-2) }}$ tumor cells, and the tumor growth was monitored twice a week for up to 65 days.

In order to investigate the therapeutic effect of the VLPs, mice (6 mice for each group) were initially challenged with $1 \times 10^{6}$ Lewis $^{(\text {Trop-2) }}$ cells subcutaneously in the right flank on day 0 . Next, on days 4 and 18 , challenged mice were inoculated with $40 \mu \mathrm{g}$ of each kind of VLPs or with $100 \mu \mathrm{l}$ PBS, respectively. Mice were monitored twice a week and the survival rate was recorded.

Total anti-Trop-2 IgG measurement. Trop-2-specific IgG titers in the mouse sera were detected by ELISA. Briefly, a 96-well plate was coated overnight at $4^{\circ} \mathrm{C}$ with purified Trop-2 protein, which was expressed and purified using Ni-NTA Agarose (Invitrogen; Thermo Fisher Scientific; cat. no. R90101) at a 

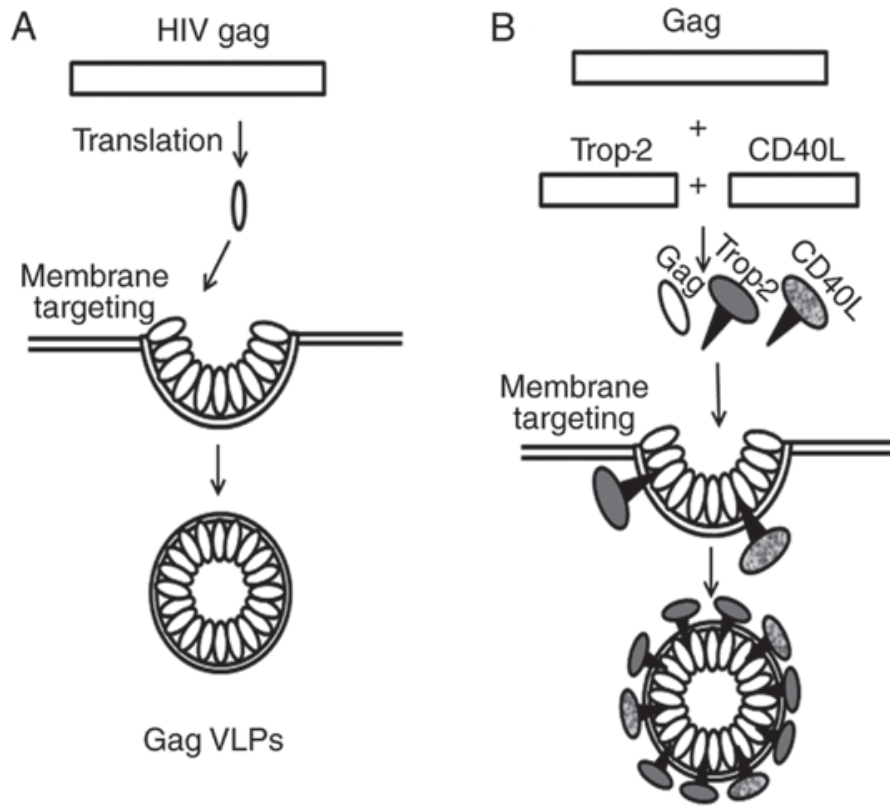

Gag VLPs

Trop-2-CD40L VLPs

Figure 1. Schematic representation of gag and Trop-2-CD40L polyprotein, and VLP formation. Representation of the formation of (A) gag VLPs and (B) Trop-2-CD40L VLPs. CD40L, CD40 ligand; VLP, virus-like particle.

concentration of $0.8 \mu \mathrm{g} /$ well. Subsequent to three washes with $200 \mu \mathrm{l}$ PBST, the plate was blocked with $100 \mu 12 \%$ bovine serum albumin for $1 \mathrm{~h}$ in a $37^{\circ} \mathrm{C}$ water bath. Following a further three washes with PBST, the serum samples were series diluted and added into the wells (100 $\mu \mathrm{l} /$ well) for incubation at $37^{\circ} \mathrm{C}$ for $1 \mathrm{~h}$. A horseradish peroxidase-conjugated goat anti-mouse $\operatorname{IgG}$ second antibody with a dilution of 1:5,000 (cat. no. ZDR-5307; Origene Technologies, Inc.) was then added. After three further washes, the color was developed using 3,3',5,5'-tetramethylbenzidine/hydrochloride (Sigma-Aldrich; Merck KGaA; cat. no. ALDR-ICH-860336), and the optical density at $450 \mathrm{~nm}$ was recorded.

Cytokine detection by ELISA. In order to detect the cytokines produced following immunization, the splenocytes of each immunized mice were isolated and mixed. Next, CD4 ${ }^{+}$ $\mathrm{T}$ cells or $\mathrm{CD}^{+} \mathrm{T}$ cells were isolated using the corresponding $\mathrm{CD}^{+} / \mathrm{CD} \mathrm{a}^{+} \mathrm{T}$ cell Isolation kit (Miltenyi Biotec $\mathrm{GmbH}$, Bergisch Gladbach, Germany). Cells were then cultured in 96-well microplates at a density of $2 \times 10^{4}$ cells/well (EMD Millipore, Billerica, MA, USA) with $100 \mu$ RPMI 1640 (Gibco; Thermo Fisher Scientific, Inc.) containing 10\% FBS and stimulated with Trop-2 peptide (ProSci, Inc., Poway, CA, USA). Subsequent to incubation at $37^{\circ} \mathrm{C}$ for $48 \mathrm{~h}$, the plate was centrifuged at $300 \mathrm{x}$ g for $5 \mathrm{~min}$, and the cell culture supernatant was carefully collected and assayed using ELISA kits for the production of interferon (IFN)- $\gamma$ (cat. no. 88-8314-88), interleukin (IL)-29 (cat. no. 88-7024-88), IL-10 (cat. no. BMS614-2) and IL-4 (cat. no. BMS613TWO) using cytokine ELISA kits (all Invitrogen; Thermo Fisher Scientific, Inc.).

Statistical analysis. Results are expressed as the mean \pm standard error of the mean, and were analyzed by one-way analysis of variance. P-values of $<0.05$ were considered to indicate differences that were statistically significant.

\section{Results}

Trop-2 and CD4OL are effectively incorporated onto VLPS. HIV gag can be modified to form type II VLPs with heterologous antigens on the surface of their membrane envelope. A model describing the formation of gag VLPs and chimeric Trop-2-CD40L VLPs is shown in Fig. 1. The morphology of VLPs was detected under an electron microscope. As shown in Fig. 2A, Trop-2 and Trop-2-CD40L VLPs exhibited a similar morphology compared with gag VLPs, with a diameter of $\sim 110 \mathrm{~nm}$. VLPs were also characterized by immunoblotting using monoclonal antibodies specifically recognizing mTrop-2, CD40L or HIV gag p24. As shown in Fig. 2B, Trop-2 protein was detected in Trop-2 and Trop-2-CD40L VLPs; however, CD40L was only expressed in Trop-2-CD40L VLPs. Therefore, these data strongly suggested that $\mathrm{rBv}$ infection successfully released mTrop-2 VLPs and mTrop-2-CD40L VLPs.

CD40L-incorporated VLPs activate B-cells. As CD40L serves a critical role in the B-cell activation process, splenocytes were cultured from naïve mice in the presence of Trop-2-CD40L or Trop-2 VLPs. After 4 days, the expression of the B-cell activation marker CD69 was assessed to analyze the $\mathrm{B}$-cell activation $(\mathrm{P}<0.01$; Fig. 3$)$. It was observed that when splenocytes were cultured with chimeric Trop-2-CD40L VLPs, increased numbers of activated $\mathrm{CD} 69^{+} \mathrm{B} 220^{+}$cells were detected, whereas less CD69 ${ }^{+} \mathrm{B} 220^{+} \mathrm{B}$-cells were detected post Trop-2 VLPs stimulation. Overall, incorporation of CD40L increased the number of $\mathrm{CD} 69^{+} \mathrm{B} 220^{+}$cells by nearly a 4 -fold.

Trop-2-CD4OL VLPs enhance anti-Trop-2 IgG production in immunized mice. ELISA was performed to determine whether Trop-2-CD40L VLP immunization was able to induce a higher level of anti-Trop-2 antibody when compared with Trop-2 VLP 


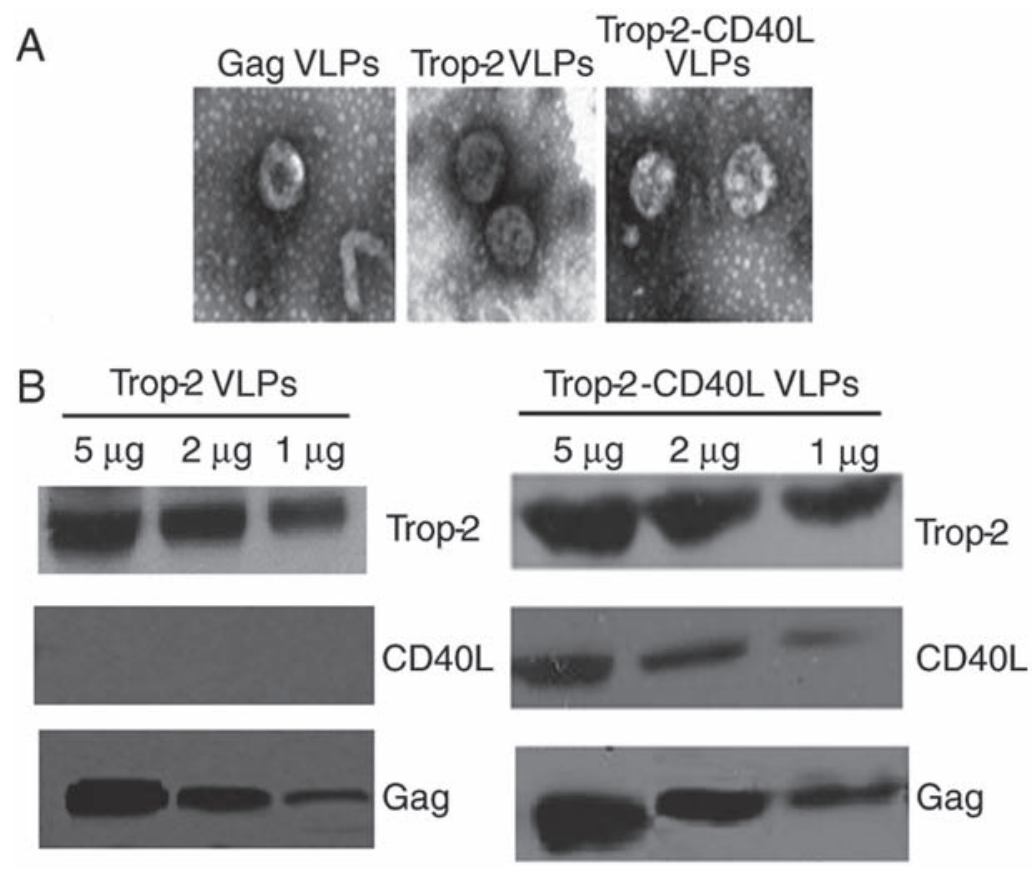

Figure 2. Characterization of VLPs. (A) Detection of VLPs under a transmission electron microscope. Purified gag, Trop-2 and Trop-2-CD40L VLPs were negatively stained with $1 \%$ uranyl acetate, washed and observed under the microscope. (B) Detection of proteins in VLPs by western blot analysis. The levels of Trop-2, CD40L and gag in different concentrations of purified Trop-2 or Trop-2-CD40L VLPs were characterized. CD40L, CD40 ligand; VLPs, virus-like particles.

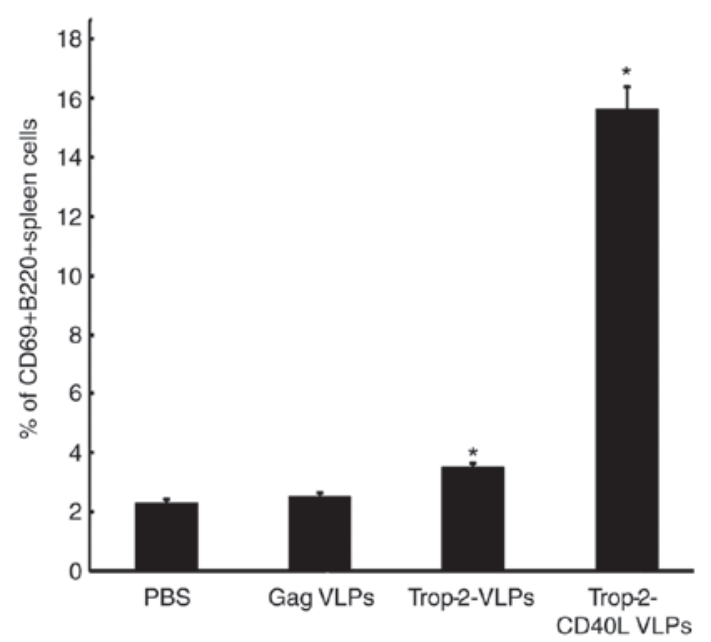

Figure 3. Activated B-cells were induced by chimeric Trop-2-CD40L VLPs. Spleen cells from naive C57BL/6 mice were isolated and then cultured with PBS or $2 \mu \mathrm{g} / \mathrm{ml}$ of each VLP in triplicate. The stimulated cells were collected on day 5 and analyzed by flow cytometry subsequent to staining with peridinin chlorophyll protein-conjugated anti-B220 and phycoerythrin-conjugated anti-CD69. The error bars denote the standard error of the mean. "P $<0.05$ compared with the PBS immunized group. CD40L, CD40 ligand; VLPs, virus-like particles.

immunization. As shown in Fig. 4, Trop-2 specific IgG was nearly undetectable in the serum of gag VLP-immunized mice. By contrast, high levels of Trop-2-specific IgG were detected from the serum of Trop-2-CD40L or Trop-2 VLP-immunized mice. Furthermore, compared with Trop-2 VLPs, Trop-2-CD40L VLPs induced a nearly 2-fold higher number of IgG titer $(\mathrm{P}<0.01)$. Therefore, incorporation of CD40L onto VLPs was able to efficiently enhance the immunogenicity of Trop-2 VLPs.

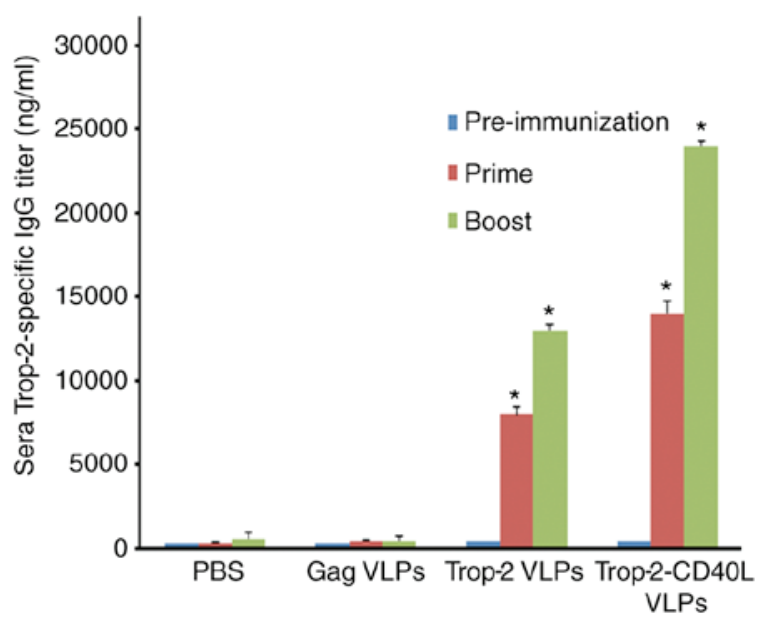

Figure 4. Chimeric Trop-2-CD40L VLPs induce higher Trop-2-specific IgG titers. C57BL/6 mice were immunized with $40 \mu \mathrm{g}$ purified gag VLPs, Trop-2 VLPs or Trop-2-CD40L VLPs at day 0 (prime immunization), respectively. Mice in all groups received a second immunization at the end of week 4 (boost immunization). Two weeks post each immunization, serum was collected and used to detect the production of Trop-2-specific IgG antibody by ELISA. "P<0.05 compared with the PBS immunized group. CD40L, CD40 ligand; VLPs, virus-like particles.

Trop-2-CD4OL VLPs enhance cellular responses more effectively. Cytokine ELISA was performed to detect the CD4 and CD8 T cell immune responses. Spleens from immunized mice were isolated, sorted and stimulated with Trop-2 peptide to measure the production of IFN- $\gamma$, IL-4, IL-2 and IL-10 by Trop-2-specific CD4 and CD8 T cells (Fig. 5). Splenocytes from Trop-2 or Trop-2-CD40L VLP-injected mice demonstrated a 4- to 6-fold decrease of IL-10-producing CD4 and CD8 $\mathrm{T}$ cells as compared with the gag VLP-immunized 


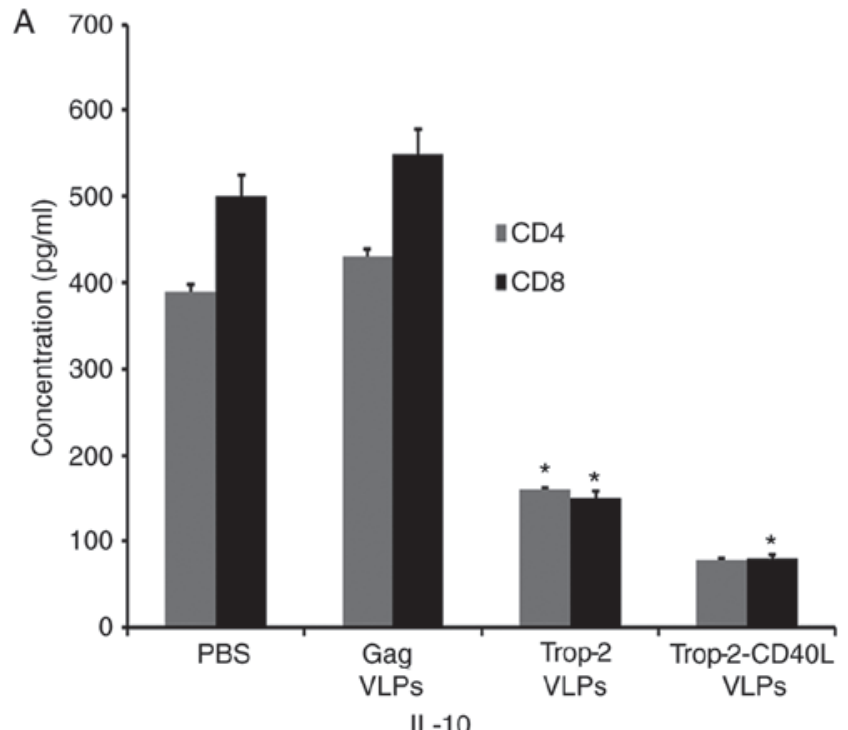

B
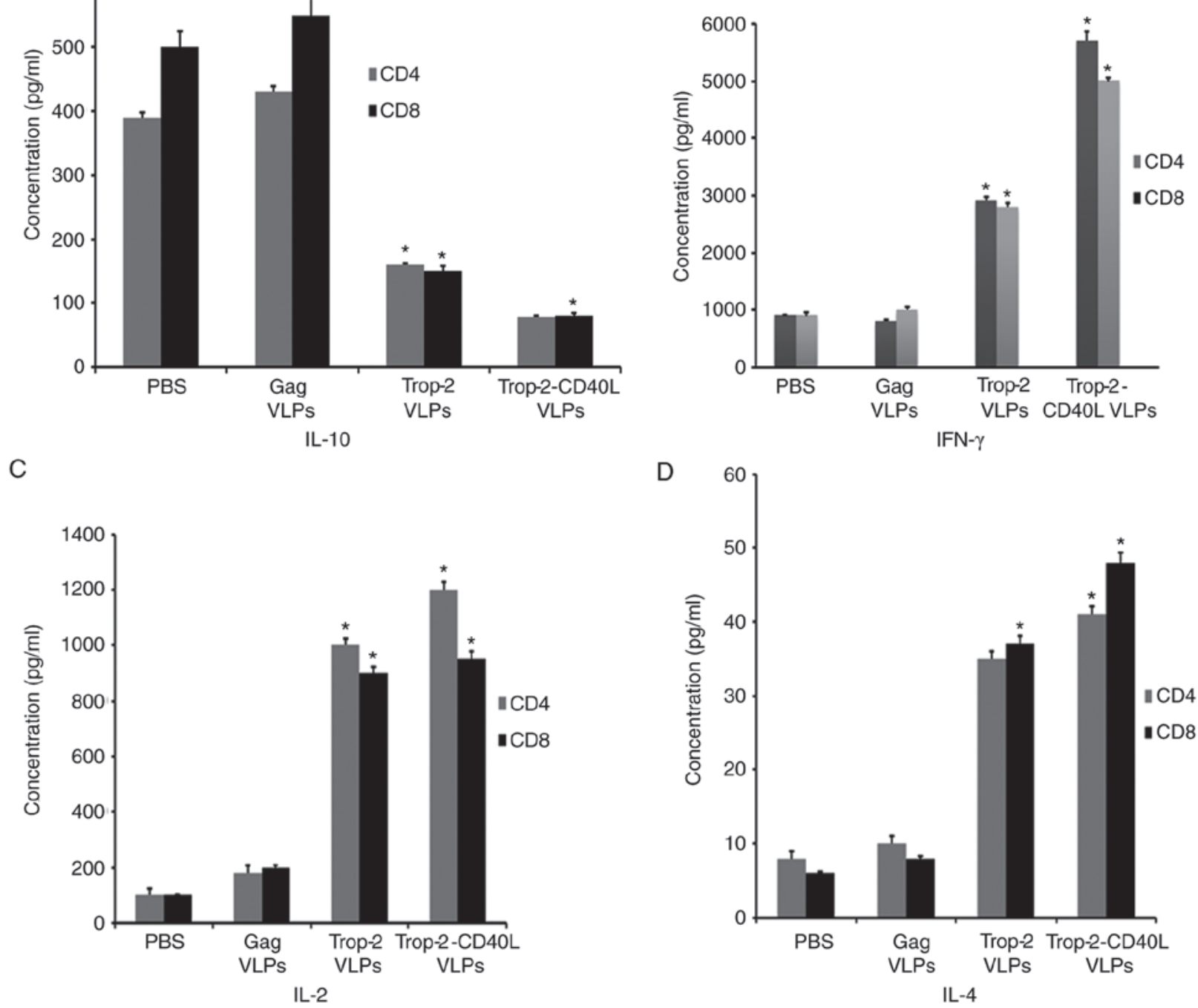

Figure 5. Trop-2-CD40L VLP immunization induced higher T-cell responses in vivo. Isolated splenocytes of each group were co-cultured with Trop-2 peptide at 4 weeks after the second immunization. The cell culture supernatant was collected after $48 \mathrm{~h}$, and cytokine ELISA was performed to measure the secretion of (A) IL-10, (B) IFN- $\gamma$, (C) IL-2, and (D) IL-4. Data are presented as the average of 5 mice in each group. "P<0.05. CD40L, CD40 ligand; VLPs, virus-like particles; IFN, interferon; IL, interleukin.

mice. The lowest IL-10 secretion level was observed in the Trop-2-CD40L VLP-immunized group (Fig. 5A). In the same experiment, the highest IFN- $\gamma$ secretion level was observed in the Trop-2-CD40L VLP-immunized group (Fig. 5B). By contrast, the Trop-2-CD40L VLP immunization exhibited a reduced effect on IL-4 and IL-2 secretion compared with the Trop-2 VLP immunization ( $\mathrm{P}=0.0008$; Fig. $5 \mathrm{C}$ and $\mathrm{D})$. These results demonstrated that incorporation of CD40L onto Trop-2 VLPs may enhance the number of IFN- $\gamma$-producing $\mathrm{T}$ cells and reduce the IL-10-producing $\mathrm{T}$ cells more potently in comparison with Trop-2 VLPs.

Preventive effect of Trop-2-CD40L VLP immunization. In vivo tumor prevention tests were conducted to determine whether immunization with Trop-2-CD40L or Trop-2 VLPs induced preventive antitumor effects against lung cancer. Female C57BL/6 mice were immunized twice with $40 \mu \mathrm{g}$ gag, Trop-2-CD40L or Trop-2 VLPs. Each mouse was then challenged with $1 \times 10^{6}$ Lewis cells and monitored for tumor formation. Fig. 6A describes a brief immunization schedule. As shown in Fig. 6B, at 65 days after the Lewis cell challenge, nearly $85 \%$ of mice immunized with Trop-2-CD40L VLPs remained tumor-free, while the percentage of tumor-free mice in the Trop-2 VLP-immunized group was $<70 \%$. However, $100 \%$ of the mice immunized with PBS or gag VLPs developed tumors $(\mathrm{P}<0.01$ compared with the PBS immunized group).

Trop-2-CD40L VLP immunization increases the survival of tumor-bearing mice more effectively. The present study also investigated whether Trop-2-CD40L or Trop-2 VLP immunization increased the survival of tumor-bearing mice. In this experiment, mice were initially challenged subcutaneously with Lewis ${ }^{(T r o p-2)}$ cells, followed by VLP injection in each corresponding group. Fig. 7A describes a brief immunization schedule. As shown in Fig. 7B, all mice in the PBS or gag VLP-immunized groups succumbed within 40 days, 


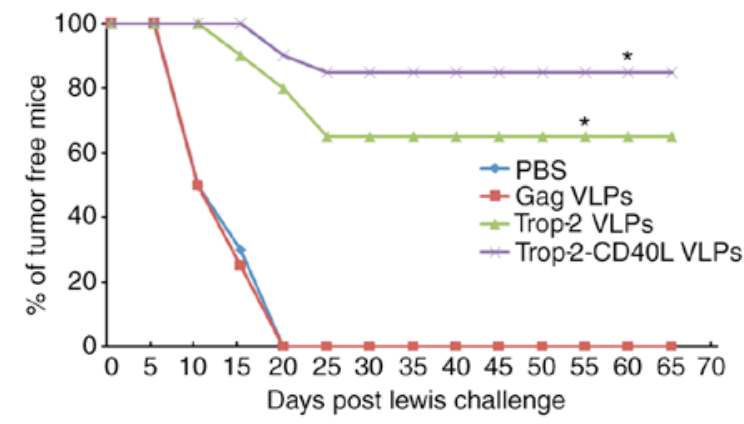

Figure 6. Trop-2-CD40L VLP injection reduced tumor growth more effectively in comparison with Trop-2 VLP immunization. (A) The immunization schedule is presented. C57BL/6 mice were immunized with $40 \mu \mathrm{g}$ purified gag VLPs, Trop-2 VLPs or Trop-2-CD40L VLPs, respectively. Mice in all groups received a boost immunization at the end of week 4 . At 1 week after the second immunization, each mouse was challenged subcutaneously with $1 \times 10^{5}$ Lewis $^{(\text {Trop-2) }}$ cells. (B) Following Lewis cell challenge, tumor growth was monitored twice a week over 65 days. In total, $85 \%$ of Trop-2-CD40L VLP-immunized mice remained tumor-free, whereas only $<70 \%$ of Trop-2 VLP-inoculated mice remained tumor-free. CD40L, CD40 ligand; VLPs, virus-like particles.
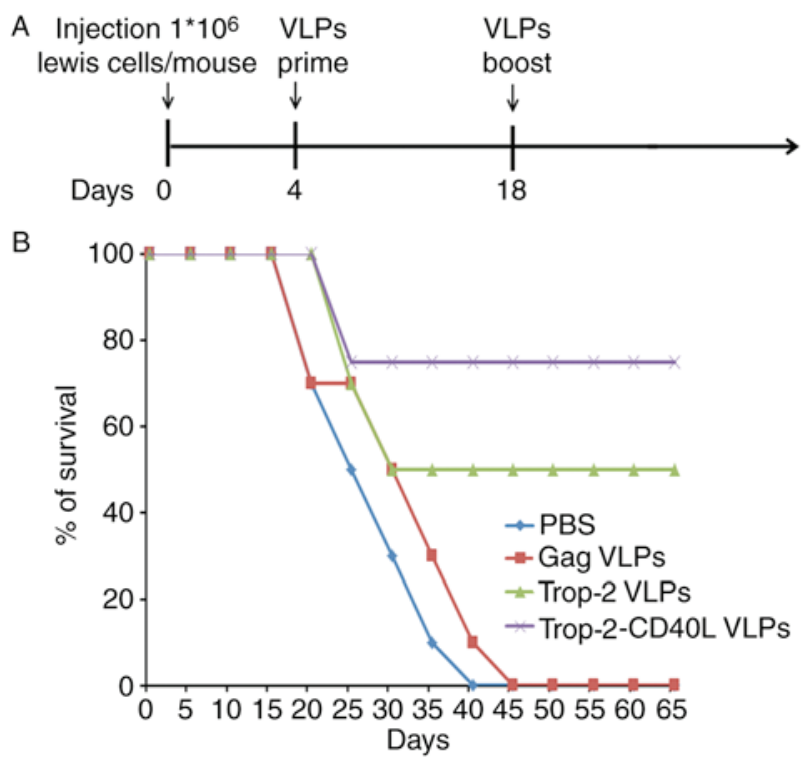

Figure 7. Trop-2-CD40L VLP injection increased the survival rate of tumor-bearing mice more effectively in comparison with Trop-2 VLP immunization. (A) C57BL/6 mice were initially inoculated with Lewis ${ }^{(\text {Trop-2) }}$ cells and then immunized twice at day 4 (prime immunization) and day 18 (boost immunization). (B) Trop-2-CD40L VLP injection demonstrated an increase in the survival (75\%) when compared with the Trop-2 VLP treatment $(50 \%)$. CD40L, CD40 ligand; VLPs, virus-like particles.

whereas the Trop-2 and Trop-2-CD40L VLP-immunized mice demonstrated significantly higher survival rates $(\mathrm{P}<0.001)$. Furthermore, the highest survival rate reached $75 \%$ in the Trop-2-CD40L VLP-immunized group.

\section{Discussion}

As the leading cause of cancer-associated mortality in China (33), lung cancer has been demonstrated to be immunogenic. Inhibitors of PD-L1 have been approved for use in the treatment of squamous and non-squamous lung cancer; however, growing populations of NSCLC patients do not respond to PD-1/PD-L1 inhibition (34). Thus, novel immunotherapy targets or immunomodulatory agents need to be explored. Trop- 2 is a recently identified cell surface glycoprotein, and has been considered to be a promising target for cancer immunotherapy. A study by Cubas et al (35) on pancreatic cancer revealed that mTrop- 2 was able to be effectively incorporated on the membrane envelope of SIV gag-based VLPs. The study also indicated that mTrop-2 VLP immunization activated humoral immune responses, as well as cellular immune response, while mTrop-2 VLPs demonstrated an improved effect when combined with the chemotherapy agent gemcitabine (35). However, whether Trop-2 may be used as an immunotherapy target against lung cancer has not yet been examined.

As a well-known immune-adjuvant, CD40L is widely used in DNA vaccine development $(36,37)$. In the field of VLPs design, CD40L was successfully incorporated into Hantaan VLPs (29) and SIV VLPs, and whether it is an effective adjuvant in lung cancer VLPs based vaccine development is yet to be determined.

In the present study, using a HIV gag backbone-based VLP strategy, Trop-2 VLPs were constructed, while the immune adjuvantCD40L was also incorporated onto the surface of the mTrop-2 VLPs. The results of the present study revealed that a HIV gag VLP-based Trop-2-CD40L vaccine was successfully prepared. Trop-2 and Trop-2-CD40L VLPs were then used to immunize C57BL/6 mice, and the serum samples of the immunized mice were examined. The Trop-2-specific IgG level was markedly increased at 2 weeks post boost immunization as measured by ELISA in the Trop-2 and Trop-2-CD40L VLP-immunized groups. In addition, the Trop-2-CD40L VLPs immunization induced a higher IgG titer in comparison with the Trop-2 VLPs immunization. Furthermore, the present study used a cytokine ELISA method to detect the cellular immune responses. The results revealed that Trop-2-CD40L VLPs immunization stimulated Trop-2-specific cellular immune responses more effectively in comparison with Trop-2 VLPs immunization, which was evidenced by the elevated IFN- $\gamma$ and reduced IL-10 secretion in Trop-2 peptide-stimulated splenocytes. This result was consistent with Lin et al's (30) study that the incorporation of CD40L into HTNV VLPs significantly enhanced their immunogenicity by enhancing cellular immune responses. In the prevention and therapeutic experiments conducted in the present study, tumor-bearing mice were prepared by Lewis ${ }^{(\text {Trop-2) }}$ injection, and tumor growth was monitored. The tumor growth was suppressed more efficiently in the Trop-2-CD40L VLP-immunized group as compared with that in the Trop-2 VLP-immunized group. Therefore, addition of the CD40L immune adjuvant onto Trop-2 VLPs provided a more powerful capacity to induce antitumor immunity.

In conclusion, the results of the present study demonstrate that immunization with either Trop-2 or Trop-2-CD40L VLPs generated specific humoral and cellular immune responses against Trop-2, whilst Trop-2-CD40L VLPs generated a higher immune response. In the prevention and therapeutic experiments, the Trop-2-CD40L VLPs exhibited a more efficient tumor inhibition effect when compared with the Trop-2 only VLPs. The results of the present study validated that Trop- 2 has the potential to be used as an immunotherapeutic target 
for lung cancer; the current result also validated the adjuvant activity of CD40L when used in VLP-based tumor vaccines. Trop-2-CD40L VLPs, due to their immunogenic properties and effects on the immune system, therefore represent a promising approach to lung cancer immunotherapy.

\section{Acknowledgements}

Not applicable.

\section{Funding}

This study was supported by grants from the China Natural Sciences Foundation (grant nos. 81572974 and 81372836) and the Shaanxi Science and Technology Innovation Project (grant no. 2016KTZDSF01-07).

\section{Availability of data and materials}

The datasets used and/or analyzed during the current study are available from the corresponding author on reasonable request.

\section{Authors' contributions}

ZHZ conceived and designed the study. DK and LM performed the animal experiments. WL performed the cell culture experiments; ZJH provided the Molecular Biology Experiments; LF performed the VLPs production and identification part; GZW and ZZ detected the antibody responses and cellular responses post immunization; WX wrote the paper. CX reviewed and edited the manuscript. All authors read and approved the manuscript.

\section{Ethics approval and consent to participate}

The present study was approved by the Institutional Review Board of Tangdu Hospital of the Fourth Military Medical University (assigned no. TDLL-201611-25).

\section{Consent for publication}

Not applicable.

\section{Competing interests}

The authors declare that they have no competing interests as defined by Molecular Medicine, or other interests that might be perceived to influence the results and discussion reported in this paper.

\section{References}

1. Fong D, Moser P, Krammel C, Gostner JM, Margreiter R, Mitterer M, Gastl G and Spizzo G: High expression of TROP2 correlates with poor prognosis in pancreatic cancer. $\mathrm{Br} J$ Cancer 99: 1290-1295, 2008.

2. Simms A, Jacob RP, Cohen C and Siddiqui MT: TROP-2 expression in papillary thyro-id carcinoma: Potential diagnostic utility. Diagn Cytopathol 44: 26-31, 2016.

3. Trerotola M, Ganguly KK, Fazli L, Fedele C, Lu H, Dutta A, Liu Q, De Angelis T, Riddell LW, Riobo NA, et al: Trop-2 is up-regulated in invasive prostate cancer and displaces FAK from focal contacts. Oncotarget 6: 14318-14328, 2015 .
4. Wang J,Day R, Dong Y, Weintraub SJ and Michel L: Identification of Trop-2 as an oncogene and an attractive therapeutic target in colon cancers. Mol Cancer Ther 7: 280-285, 2008.

5. Stepan LP, Trueblood ES, Hale K, Babcook J, Borges L and Sutherland CL: Expression of Trop-2 cell surface glycoprotein in normal and tumor tissues: Potential implications as a cancer therapeutic target. J Histochem Cytochem 59: 701-710, 2011.

6. Ambrogi F, Fornili M, Boracchi P, Trerotola M, Relli V, Simeone P, La Sorda R, Lattanzio R, Querzoli P, Pedriali M, et al: Trop-2 is a determinant of breast cancer survival. PLoS One 9: e96993, 2014.

7. Bignotti E, Zanotti L, Calza S, Falchetti M, Lonardi S, Ravaggi A, Romani C, Todeschini P, Bandiera E, Tassi RA, et al: Trop-2 protein overexpression is an indepen-dent marker for predicting disease recurrence in endometrioid endometrial carcinoma. BMC Clin Pathol 12: 22, 2012.

8. Bignotti E, Todeschini P, Calza S, Falchetti M, Ravanini M, Tassi RA, Ravaggi A, Bandiera E, Romani C, Zanotti L, et al: Trop-2 overexpression as an independent marker for poor overall survival in ovarian carcinoma patients. Eur J Cancer 46: 944-953, 2010.

9. Jiang A, Gao X, Zhang D, Zhang L and Lu H: Expression and clinical significance of the Trop-2 gene in advanced non-small cell lung carcinoma. Oncol Lett 6: 375-380, 2013.

10. Pushko P, Tretyakova I, Hidajat R, Zsak A, Chrzastek K, Tumpey TM and Kapczynski DR: Virus-like particles displaying $\mathrm{H} 5, \mathrm{H} 7, \mathrm{H} 9$ hemagglutinins and $\mathrm{N} 1$ neuraminidase elicit protective immunity to heterologous avian influenza viruses in chickens. Virology 501: 176-182, 2017.

11. Hill BD, Zak A, Khera E and Wen F: Engineering virus-like particles for antigen and drug delivery. Curr Protein Pept Sci 19: 112-127, 2018.

12. Zdanowicz M and Chroboczek J: Virus-like particles as drug delivery vectors. Acta Biochim Pol 63: 469-473, 2016.

13. Liu X, Fang Y, Zhou P, Lu Y, Zhang Q, Xiao S, Dong Z, Pan L, Lv J, Zhang Z, et al: Chimeric virus-like particles elicit protective immunity against serotype $\mathrm{O}$ foot-and-mouth disease virus in guinea pigs. Appl Microbiol Biotechnol 101: 4905-4914, 2017.

14. Jeong $\mathrm{H}$ and Seong BL: Exploiting virus-like particles as innovative vaccines against emerging viral infections. J Microbiol 55: 220-230, 2017.

15. Mohan T, Berman Z, Luo Y, Wang C, Wang S, Compans RW and Wang BZ: Chimeric virus-like particles containing influenza HA antigen and GPI-CCL28 induce long-lasting mucosal immunity against H3N2 viruses. Sci Rep 7: 40226, 2017.

16. Robert MA, Lytvyn V, Deforet F, Gilbert R and Gaillet B: Virus-like particles derived from HIV-1 for delivery of duclear proteins: Improvement of production and activity by protein engineering. Mol Biotechnol 59: 9-23, 2017.

17. Feng Q, He Y and Lu J: Virus-like larticles lroduced in lichia lastoris induce protective immune responses against Coxsackievirus A16 in mice. Med Sci Monit 22: 3370-3382, 2016.

18. Yan Q, Wu L, Chen L, Qin Y, Pan Z and Chen M: Vesicular stomatitis virus-based vaccines expressing EV71 virus-like particles elicit strong immune responses and protect newborn mice from lethal challenges. Vaccine 34: 4196-4204, 2016.

19. Wang X, Xiao X, Zhao M, Liu W, Pang L, Sun X, Cen S, Yang BB, Huang Y, Sheng W and Zeng Y: EV71 virus-like particles produced by co-expression of capsid proteins in yeasT cells elicit humoral protective response against EV71 lethal challenge. BMC Res Notes 9: 42, 2016.

20. Alshaikhahmed K and Roy P: Generation of virus-like particles for emerging epizootic haemorrhagic disease virus: Towards the development of safe vaccine candidates. Vaccine 34: 1103-1108, 2016.

21. Ludwig C and Wagner R: Virus-like particles-universal molecular toolboxes. Curr Opin Biotechnol 18: 537-545, 2007.

22. Deml L, Speth C, Dierich MP, Wolf $\mathrm{H}$ and Wagner R: Recombinant HIV-1 Pr55gag virus-like particles: Potent stimulators of innate and acquired immune responses. Mol Immunol 42: 259-277, 2005.

23. Figgett WA, Vincent FB, Saulep-Easton D and Mackay F: Roles of ligands from the TNF superfamily in B cell development, function, and regulation. Semin Immunol 26: 191-202, 2014.

24. Bazzoni $\mathrm{F}$ and Beutler B: The tumor necrosis factor ligand and receptor families. N Engl J Med 334: 1717-1725, 1996.

25. Gupta S, Termini JM, Kanagavelu S and Stone GW: Design of vaccine adjuvants incorporating TNF superfamily ligands and TNF superfamily molecular mimics. Immunol Res 57: 303-310, 2013. 
26. Song I, Kim J, Kwon K, Koo S and Jo D: Expression of CD154 (CD40L) on stimulated T lymphocytes in patients with idopathic thrombocytopenic purpura. Hematology 21: 187-192, 2016.

27. Quezada SA, Jarvinen LZ, Lind EF and Noelle RJ: CD40/CD154 interactions at the interface of tolerance and immunity. Annu Rev Immunol 22: 307-328, 2004.

28. Tripp RA, Jones L, Anderson LJ and Brown MP: CD40 ligand (CD154) enhances the Th1 and antibody responses to respiratory syncytial virus in the C57BL/6 mouse. J Immunol 164 5913-5921, 2000.

29. Cheng LF, Wang F, Zhang L, Yu L, Ye W, Liu ZY, Ying QK, $\mathrm{Wu} \mathrm{XA}, \mathrm{Xu} \mathrm{ZK}$ and Zhang FL: Incorporation of GM-CSF or CD40L enhances the immunogenicity of Hantaan virus-like particles. FronT cell Infect Microbiol 6: 185, 2016

30. Stone GW, Barzee S, Snarsky V, Kee K, Spina CA, Yu XF and Kornbluth RS: Multimeric soluble CD40 ligand and GITR ligand as adjuvants for human immunodeficiency virus DNA vaccines. J Virol 80: 1762-1772, 2006.

31. Skountzou I, Quan FS, Gangadhara S, Ye L, Vzorov A, Selvaraj P, Jacob J, Compans RW and Kang SM: Incorporation of glycosylphosphatidylinositol-anchored granulocyte-macrophage colony-stimulating factor or CD40 ligand enhances immunogenicity of chimeric simian immunodeficiency virus-like particles. J Virol 81: 1083-1094, 2007.
32. Daniel RA and Errington J: Control of cell morphogenesis in bacteria: two distinct ways to make a rod-shaped cell. Cell 113: 767-776, 2003

33. Chen W, Zheng R, Zeng $\mathrm{H}$ and Zhang S: Epidemiology of lung cancer in China. Thorac Cancer 6: 209-215, 2015.

34. Somasundaram A and Burns TF: The next generation of immunotherapy: Keeping lung cancer in check. J Hematol Oncol 10: 87, 2017.

35. Cubas R, Zhang S, Li M, Chen C and Yao Q: Chimeric Trop-2 virus-like particles: A potential immunotherapeutic approach against pancreatic cancer. J Immunother 34: 251-263, 2011.

36. Wang Y, Wang YM, Wang Y, Zheng G, Zhang GY, Zhou JJ, Tan TK, Cao Q, Hu M, Watson D, et al: DNA vaccine encoding CD40 targeted to dendritic cells in situ prevents the development of Heymann nephritis in rats. Kidney Int 83: 223-232, 2013.

37. Kwa S, Sadagopal S, Shen X, Hong JJ, Gangadhara S, Basu R, Victor B, Iyer SS, LaBranche CC, Montefiori DC, et al: CD40L-adjuvanted DNA/modified vaccinia virus Ankara simian immunodeficiency virus (SIV) vaccine enhances protection against neutralization-resistant mucosal SIV infection. J Virol 89: 4690-4695, 2015. 